\title{
Three-dimension versus two-dimension video-assisted thoracoscopic surgery for esophageal cancer: a meta-analysis
}

\author{
Ning Xin^, Xinyu Ding, Kenan Huang, Rongqiang Wei, Zihao Chen, Chengdong Liu, Yunhao Fang, \\ Zhifei Xu, Hua Tang \\ Department of Thoracic Surgery, Shanghai Changzheng Hospital, Navy Military Medical University, Shanghai, China \\ Contributions: (I) Conception and design: H Tang; (II) Administrative support: Z Xu; (III) Provision of study materials or patients: X Ding, K Huang, \\ R Wei; (IV) Collection and assembly of data: Z Chen, C Liu, Y Fang; (V) Data analysis and interpretation: N Xin; (VI) Manuscript writing: all \\ authors; (VII) Final approval of manuscript: all authors. \\ Correspondence to: Hua Tang, MD. Department of Thoracic Surgery, Shanghai Changzheng Hospital, Navy Military Medical University, No.415, \\ Fengyang Road, Huangpu District, Shanghai 20001, China. Email: tangh_mits@163.com.
}

Background: It still remains unclear whether three-dimension (3D) video-assisted thoracoscopic surgery (VATS) for esophageal cancer is safe and reasonable. This meta-analysis aims at assessing the effectiveness and safety of 3D VATS for esophageal cancer in comparison with that of two-dimension (2D) VATS.

Methods: All the relevant data systematically analyzed in this thesis is from PubMed, Embase, The Cochrane Library, Web of Science and clinicaltrials.gov, and the time span for retrieval is from the date of the database establishment to February 2021. The research on the efficacy and safety of 3D VATS for esophageal cancer and 2D VATS is consistent with our meta-analysis. Continuous variables and dichotomy variables are compared using odds ratio, average or standard average differences with $95 \%$ confidence interval (95\% CI), and $\mathrm{P}$ values, respectively.

Results: In five studies of this paper, there were 553 patients in total (3D VATS group, n=266 and 2D VATS group, $\mathrm{n}=287$ ). Patients in the $3 \mathrm{D}$ group had shorter operation time [standardized mean difference $(\mathrm{SMD})=-0.99,95 \% \mathrm{CI}:-1.66$ to $-0.32 ; \mathrm{P}=0.004]$, and less bleeding $(\mathrm{SMD}=-0.88,95 \% \mathrm{CI}:-1.66$ to $-0.10 ; \mathrm{P}=0.03$ ) than those in the $2 \mathrm{D}$ group. The total amount of dissected lymph node and post-operative complications in the 2D group and the $3 \mathrm{D}$ group were nearly the same, showing no significant difference.

Discussion: The results of this meta-analysis showed that 3D VATS for esophageal cancer will be more applied and developed in the future.

Registration number of PROSPERO: CRD42021238863.

Keywords: Three-dimension (3D); two-dimension (2D); video-assisted thoracoscopic surgery (VATS); esophageal cancer

Submitted Apr 13, 2021. Accepted for publication May 21, 2021.

doi: $10.21037 /$ tcr-21-644

View this article at: https://dx.doi.org/10.21037/tcr-21-644

\section{Introduction}

Despite the fact that esophagectomy with extended lymph node dissection has always been regarded as one of the most invasive gastrointestinal surgeries $(1,2)$, esophagectomy is still the mainstream treatment of localized esophageal cancer (3-6). Since 1992 when endoscopic esophagectomy was proposed by Cuschieri (7) to cure patients with esophageal cancer, minimally invasive surgery for esophageal cancer has been advanced gradually and steadily. In recent years, minimally invasive esophagectomy (MIE), as a less invasive surgical method, has been more and more used in the treatment of esophageal cancer, which is

^ ORCID: 0000-0002-7348-7919. 


\begin{tabular}{|c|c|c|c|c|c|}
\hline Search & Actions & Details & Query & Results & Time \\
\hline$\# 9$ & $\cdots$ & $>$ & $\begin{array}{l}\text { Search: (((Esophageal Neoplasms[MeSH]) OR (Esophag* Neoplasm* } \\
\text { [Title/Abstract])) OR (Esophag* Cancer*[Title/Abstract])) AND } \\
\text { (((Imaging, Three-Dimensional[MeSH]) OR (3D[Title/Abstract])) OR } \\
\text { (3-D[Title/Abstract])) }\end{array}$ & 576 & $08: 14: 46$ \\
\hline$\# 8$ & $\cdots$ & $>$ & $\begin{array}{l}\text { Search: ((Imaging, Three-Dimensional[MeSH]) OR } \\
\text { (3D[Title/Abstract])) OR (3-D[Title/Abstract]) }\end{array}$ & 258,832 & $08: 14: 34$ \\
\hline \#7 & $\cdots$ & $>$ & Search: 3-D[Title/Abstract] & 40,659 & $08: 14: 20$ \\
\hline \#6 & $\cdots$ & $>$ & Search: 3D[Title/Abstract] & 196,295 & 08:14:06 \\
\hline \#5 & $\cdots$ & $>$ & Search: Imaging, Three-Dimensional[MeSH] & 82,918 & 08:13:49 \\
\hline$\# 4$ & $\cdots$ & $>$ & $\begin{array}{l}\text { Search: ((Esophageal Neoplasms[MeSH]) OR (Esophag* Neoplasm* } \\
\text { [Title/Abstract])) OR (Esophag* Cancer*[Title/Abstract]) }\end{array}$ & 71,757 & $08: 13: 22$ \\
\hline \#3 & $\cdots$ & $>$ & Search: Esophag* Cancer*[Title/Abstract] & 48,011 & $08: 12: 26$ \\
\hline \#2 & $\cdots$ & $>$ & Search: Esophag* Neoplasm*[Title/Abstract] & 5,915 & 08:12:07 \\
\hline \#1 & $\cdots$ & $>$ & Search: Esophageal Neoplasms[MeSH] & 51,754 & 08:10:45 \\
\hline
\end{tabular}

Figure 1 Retrieval strategy.

composed of thoracoscopy and laparoscopy $(2,8)$.

With minimally invasive technique being introduced into the esophageal cancer operation, the incidence of postoperative complications will decline, while the quality of surgical resection is maintained (8-11). Nevertheless, the performance of two-dimension (2D) video-assisted thoracoscopic surgery (VATS) for esophageal cancer surgery is far from perfect. 2D VATS for esophageal cancer is limited by a restricted operating field and disturbed eyehand coordination, and so on (12).

Three-dimension (3D) VATS can overcome the above shortcomings of 2D VATS, it can provide 24 times 3D imaging and provide surgeons with deep intuition comparable to robotic systems (12). However, a prospective randomized controlled trial (RCT) of gastric cancer shows that 3D VATS laparoscopic gastrectomy does not reduce operation time more than 2D VATS, but it provides the benefit of less intraoperative bleeding than 2D VATS (13). In contrast, another prospective randomized trial has shown that 3D VATS laparoscopy does not have any benefit for oophorectomy. What's more, studies have shown that the 3D VATS may cause more frequent adverse reactions such as nausea, dizziness, ocular fatigue and blurring of vision to surgeons (14). These results show that 3D VATS does not have the same advantage in all types of endoscopic surgery. There is no clear evidence-based medical evidence to explore that 3D VATS for esophageal cancer outweighs 2D VATS in scope and magnitude (12). Hence, the current meta-analysis was intended to assess the safety and efficacy of 3D VATS for esophageal cancer in comparison with that of traditional 2D VATS.

We present the following article in accordance with the PRISMA reporting checklist (15) (available at https://dx.doi. org/10.21037/tcr-21-644).

\section{Methods}

We registered this meta-analysis in PROSPERO for the registration number is CRD42021238863.

\section{Literature search strategy}

All the documents were retrieved from PubMed, Web of Science, The Cochrane Library, Embase and clinicaltrials. gov, and the time span for data retrieval is from the date of the database establishment to February 2021. In the process of retrieving data, the method of combining subject words and free words was adopted, and the references in the part of the literature review were traced so as to complement and acquire more relevant information. For the convenience of recalling relevant documents, the retrieval strategy adopted by us is in Figure 1 .

\section{Criteria for inclusion and exclusion}

Prior to the collection of these documents, the eligibility 
criteria used in meta-analysis were decided as follows: (I) to assess the clinical safety and efficacy of using 3D VATS and 2D VATS as a therapeutic modality for esophageal cancer; (II) publish research in English or in Chinese; (III) data for at least one statistical result, including operation time, operative blood loss, total number of dissected lymph node and post-operative complications. If the study result is case reports, conference summaries, editorials, reviews or expert opinions, the data collected is not adequate for further meta-analysis, so it is not analyzed in this meta-analysis. Additionally, if there are overlapping patients in these studies, the ones with a larger sample size are selected.

\section{Study selection}

Two investigators (K Huang and R Wei) screened all the studies we collected from the database, and removed unrelated studies through viewing the title and abstract of the studies. Then we downloaded the full texts if the study was related to our meta-analysis theme. Full texts were also downloaded for further reading if we couldn't determine whether the study was related to our research theme. The screening of full texts was conducted by two investigators independently based on the selection criteria as follows: (I) study type: cohort studies (including prospective cohort studies as well as retrospective cohort studies); (II) outcome indicators: studies containing operation time, operative blood loss, total number of lymph node retrieve and postoperative complications. To increase the comprehensiveness of the study and lessen the likelihood of omitting data related to the study, preliminary selection and a full-text investigation of the cited cases were performed. If there is a disagreement between the two evaluators, the third evaluator (X Ding) decides. Risk of bias is shown in Figures 2,3.

\section{Data extraction}

Data extraction was independently performed by two investigators ( $\mathrm{Z}$ Chen and Y Fang) using the following extraction methods: (I) article information: name of the first author, publication year, research type, and country of publication; (II) information about patients: the number of patients in each group, age, gender, tumor location, TNM staging; (III) outcome parameters: operation time; secondary outcome parameters: operative blood loss, total number of lymph node retrieve and postoperative complications. A third investigator (C Liu) checked the consistency of extracted data and then reviewed the original literature for eliminating any inconsistency in data.

\section{Quality assessment}

The quality of each cohort study is assessed by using the Newcastle-Ottawa Scale (NOS) (16). The NOS in these studies consists of 3 perspectives with a maximum of 9 stars: a maximum of 4 stars for selection, 2 stars for comparability and 3 stars for the ascertainment of outcome of interest. Studies with a score of 5 or more stars were regarded as ones of high quality.

\section{Statistical analysis}

In statistical analyses, odds ratios (ORs) were used for dichotomous variables, and mean differences (MD) or standardized mean differences (SMDs) was used for continuous variables with their $95 \%$ confidence intervals (CIs). In accordance with the summarized statistics, a $\mathrm{P}$ value $<0.05$ indicated that the results had statistical significance. With regard to the literature only with the median and upper and lower limits, "mean variance estimation" was used to estimate the mean and standard deviation according to Luo et al. (17). We employed the $\chi^{2}$ based Q-statistic test and $\mathrm{I}^{2}$ test to evaluate the statistical heterogeneity. $\mathrm{I}^{2}<50 \%$ and $\mathrm{P}$ value $>0.10$ meant acceptable heterogeneity and then a fixed-effect model was applied. By contrast, if there was significant heterogeneity, a randomeffect model was used. Given the fact that the number of studies included in this paper was limited, we did not make sensitivity analysis and publication bias. Review Manager V.5.3 was used to conduct all statistical analyses.

\section{Results}

\section{Study characteristics}

From 4 databases (PubMed $n=576$, Embase $n=1,149$, The Cochrane Library $n=74$, Web of Science $n=1,012$ ) and clinicaltrials.gov $(\mathrm{n}=1), 2,812$ studies were retrieved. After preliminary screening, 1,080 articles were excluded and 1,732 studies were downloaded. A further selection of the 1,732 studies eliminated 1,722 studies with only 10 studies left. Then full-text retrieval was conducted on the remaining studies, 5 of which were excluded because of inconformity with the selection criteria. Figure 4 illustrates the literature screening process. Baseline information 


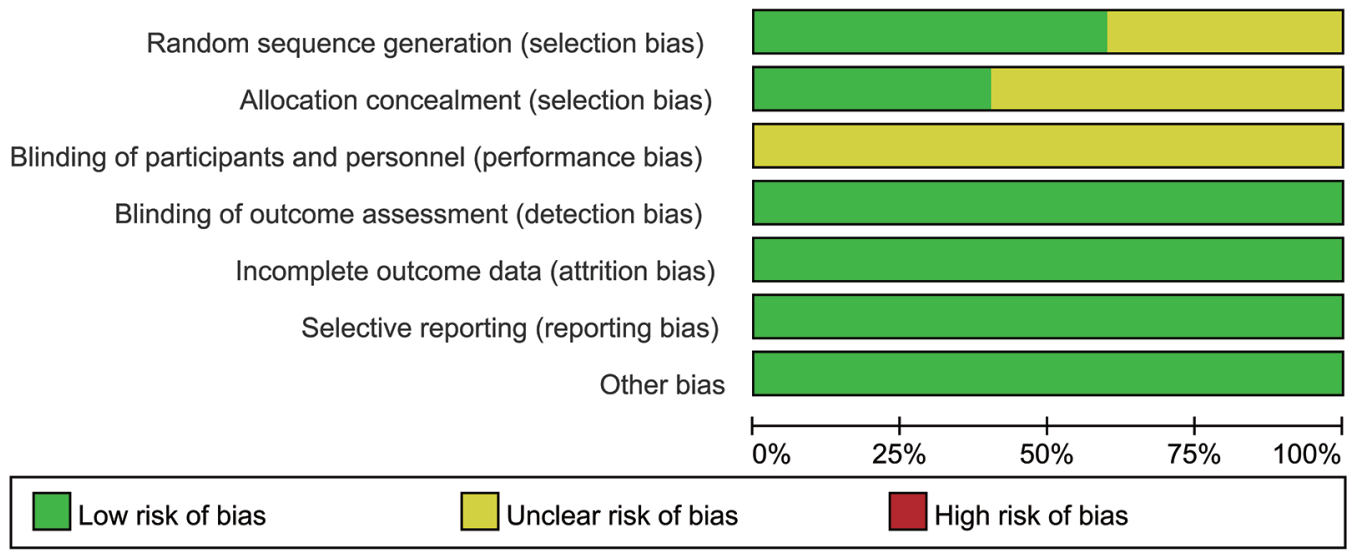

Figure 2 Risk of bias graph according to the Cochrane collaboration's tool.

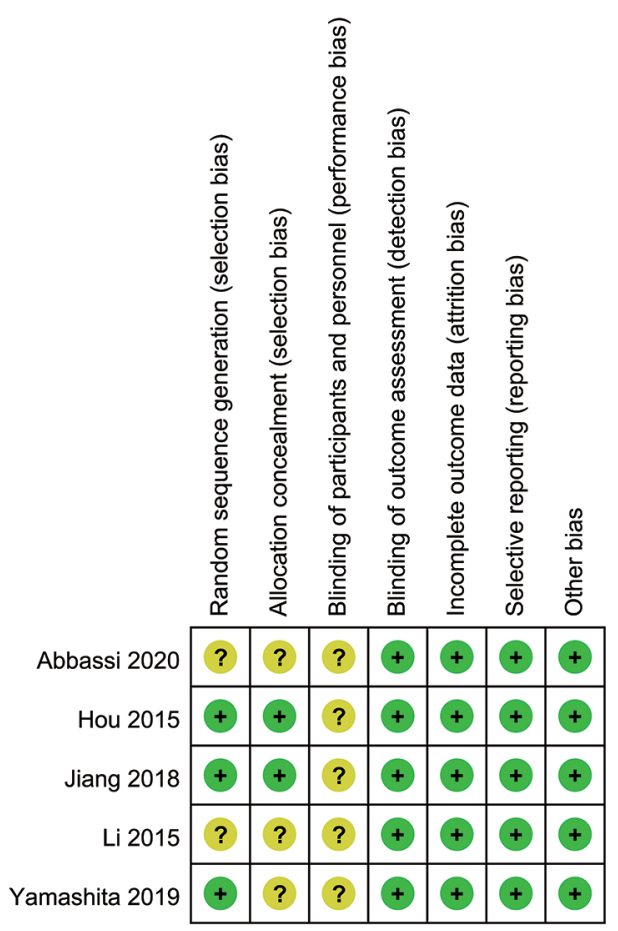

Figure 3 Risk of bias summary according to the Cochrane collaboration's tool.

collection and quality assessment were conducted on the 5 studies we selected, all of which were assessed as high quality. They were eventually selected for use in our research. Table 1 (18-21) indicates the quality assessment and baseline information of the 5 studies.

\section{Operation time}

In all studies above, the operation time of 553 patients was compared, showing significant heterogeneity between the 3D VATS group and 2D VATS group $\left(\mathrm{P}<0.001 ; \mathrm{I}^{2}=93 \%\right)$. Accordingly, the random-effects model was adopted to collect data. As is suggested by the statistics, 3D VATS took a significantly shorter operation time than 2D VATS $(\mathrm{SMD}=-0.99,95 \%$ CI: -1.66 to $-0.32 ; \mathrm{P}=0.004)$ (Figure 5$)$.

\section{Operative blood loss}

This meta-analysis contained comparable data on operative blood loss in all the 553 participants. Because there was high heterogeneity between the 3D VATS group and 2D VATS group $\left(\mathrm{P}<0.001 ; \mathrm{I}^{2}=95 \%\right)$, a random-effects model came into use. Operative blood loss between the 3D VATS group and the 2D VATS group displayed significant difference ( $\mathrm{SMD}=-0.88,95 \% \mathrm{CI}:-1.66$ to $-0.10 ; \mathrm{P}=0.03$ ) (Figure 6).

\section{Total number of lymph node retrieve}

Due to high heterogeneity between studies $(\mathrm{P}<0.001$; $\left.\mathrm{I}^{2}=82 \%\right)$, we employed a random-effects model, which showed that the number of lymph node retrieve was unrelated to 3D VATS and 2D VATS (SMD $=0.24,95 \%$ CI: -0.16 to $0.65 ; \mathrm{P}=0.24$ ) (Figure 7 ).

\section{Postoperative complications}

All the 553 subjects were included to assess the relevance 


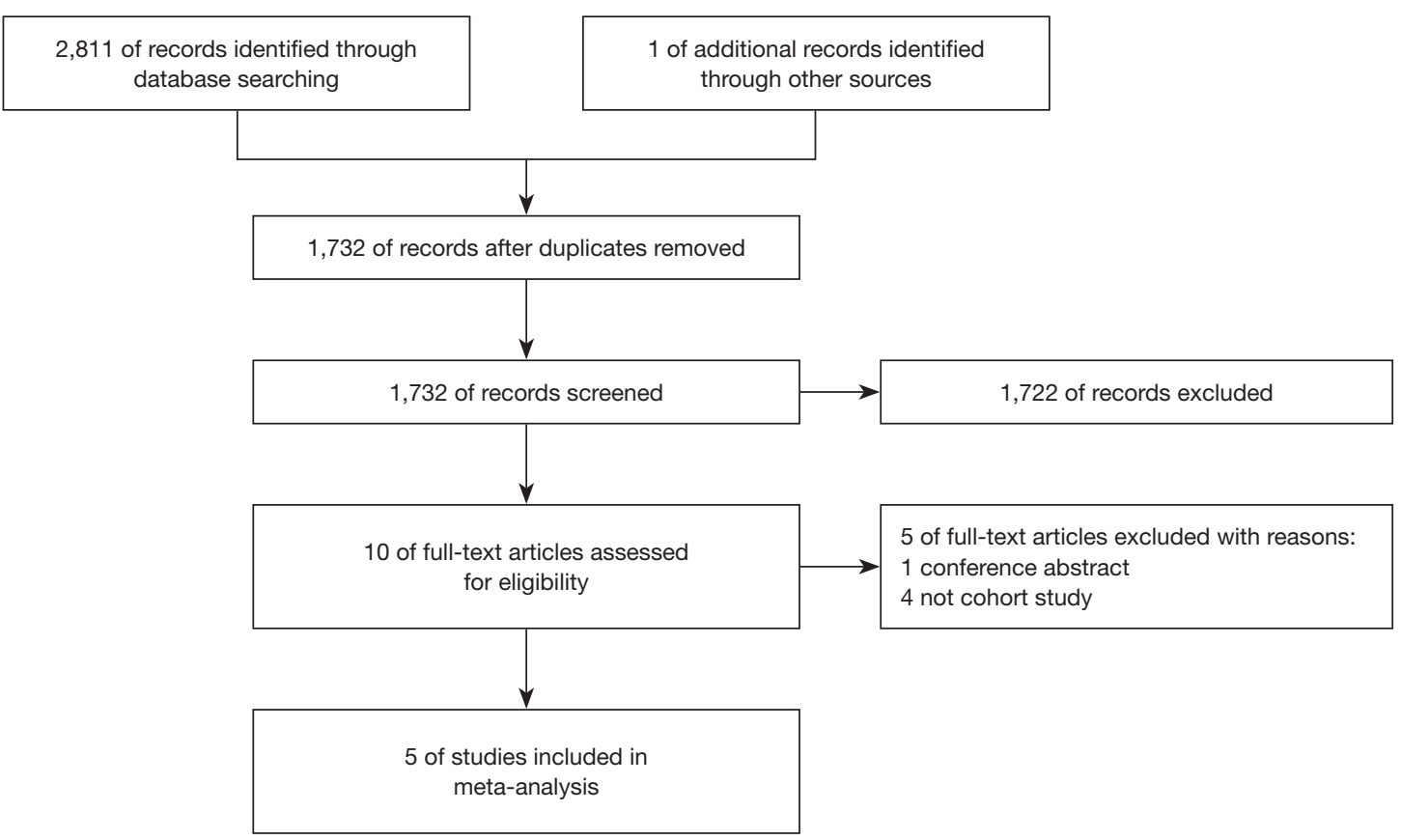

Figure 4 Preferred reporting items for meta-analyses flow chart of literature selection.

Table 1 Baseline information of included articles

\begin{tabular}{|c|c|c|c|c|c|c|c|}
\hline First author & Publication year & Ethnicity & $\begin{array}{l}\text { Number of patients } \\
\text { (3D/2D) }\end{array}$ & Age (years) (3D/2D) & \multicolumn{2}{|c|}{ Tumor level (U/M/L) } & $\begin{array}{c}\text { Quality } \\
\text { assessment }\end{array}$ \\
\hline Hou (18) & 2015 & China & $78 / 76$ & $55.7 \pm 6.3 / 55.1 \pm 7.6$ & $11 / 38 / 29$ & $21 / 32 / 23$ & NOS 6 \\
\hline Li (12) & 2015 & China & $45 / 48$ & $63.8 \pm 10.2 / 65.1 \pm 9.8$ & $8 / 29 / 8$ & $9 / 30 / 9$ & NOS 6 \\
\hline Jiang (19) & 2018 & China & $53 / 51$ & NS & NS & NS & NOS 5 \\
\hline Abbassi (21) & 2020 & UK & $39 / 59$ & $68.1 \pm 8.2 / 67.5 \pm 7.8$ & $0 / 0 / 39$ & $0 / 1 / 58$ & NOS 6 \\
\hline
\end{tabular}

U/M/L, up/middle/low; NS, not shown; NOS, Newcastle-Ottawa Scale.

between 3D VATS and 2D VATS with regard to the incidence of postoperative complications. Since there is no evidence showing significant heterogeneity $(\mathrm{P}=0.70$; $\mathrm{I}^{2}=0 \%$, a fixed-effects model was adopted. According to the statistics, the difference in postoperative complications between the 3D VATS and the 2D VATS groups was not significant $(\mathrm{OR}=1.24,95 \% \mathrm{CI}$ : 0.85 to $1.82 ; \mathrm{P}=0.26)$ (Figure 8).

\section{Discussion}

Recently, with the advances in surgical technology and the renewal of therapy concept, VATS has been widely employed in radical resection of esophageal cancer. Although the 2D VATS has been globally applied to the thoracoscopic esophagectomy, the use of the 2D VATS merely involves pressing the $3 \mathrm{D}$ figure on the plane display. In comparison to traditional open surgery, 2D VATS for esophageal cancer have two major limitations listed below (I) without a 3D view, surgeons are not able to derive depth and complicate positioning, and (II) the limited degrees of freedom of the conventional $30^{\circ}$ camera, which impairs the surgeons' dexterity (22-25). The operation has a certain degree of technical difficulty, 


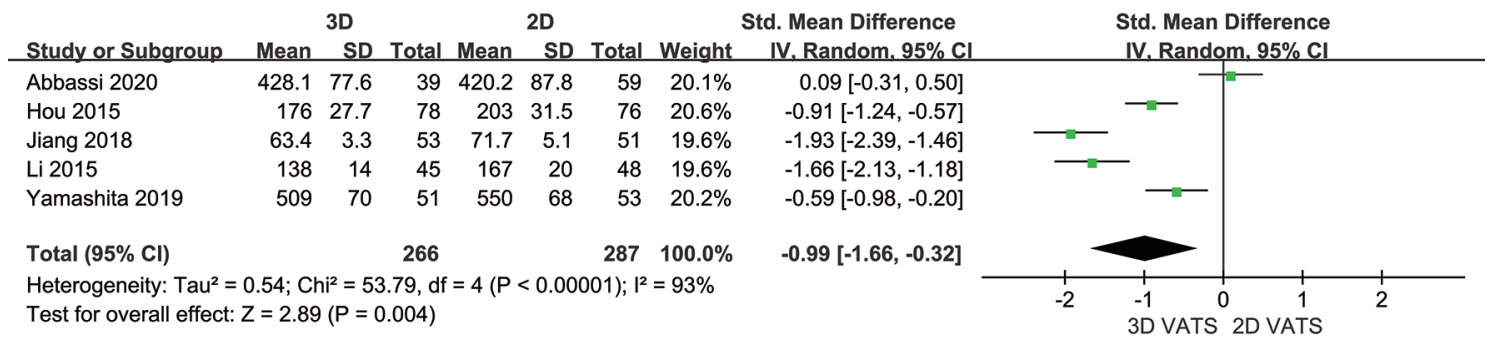

Figure 5 Forest plot of the role of SMD and its 95\% CI of operation time between 3D and 2D VATS group. SMD, standardized mean difference; CI, confidence interval; VATS, video-assisted thoracic surgery.

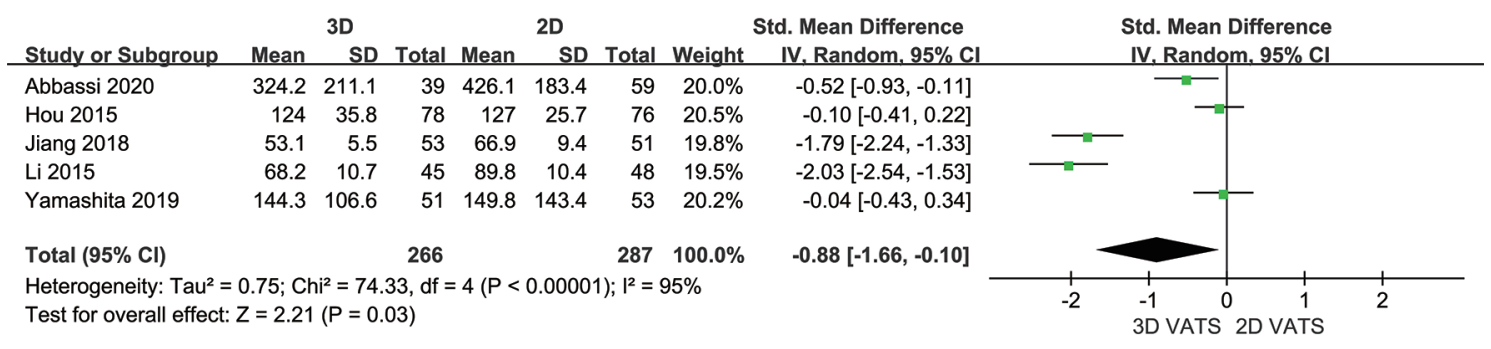

Figure 6 Forest plot of the role of SMD and its 95\% CI of operative blood loss between 3D and 2D VATS group. SMD, standardized mean difference; CI, confidence interval; VATS, video-assisted thoracic surgery.

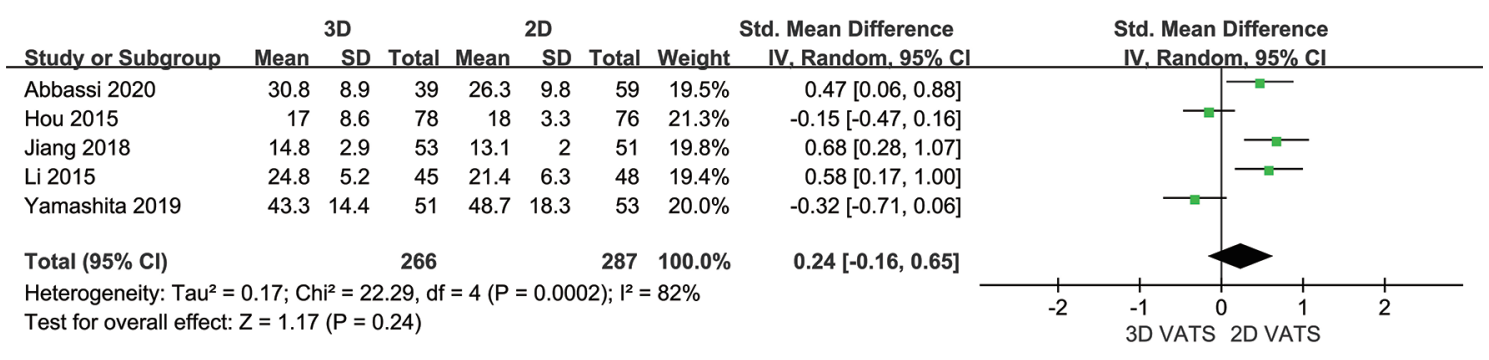

Figure 7 Forest plot of the role of SMD and its 95\% CI of Total number of lymph node retrieve between 3D and 2D VATS group. SMD, standardized mean difference; CI, confidence interval; VATS, video-assisted thoracic surgery.

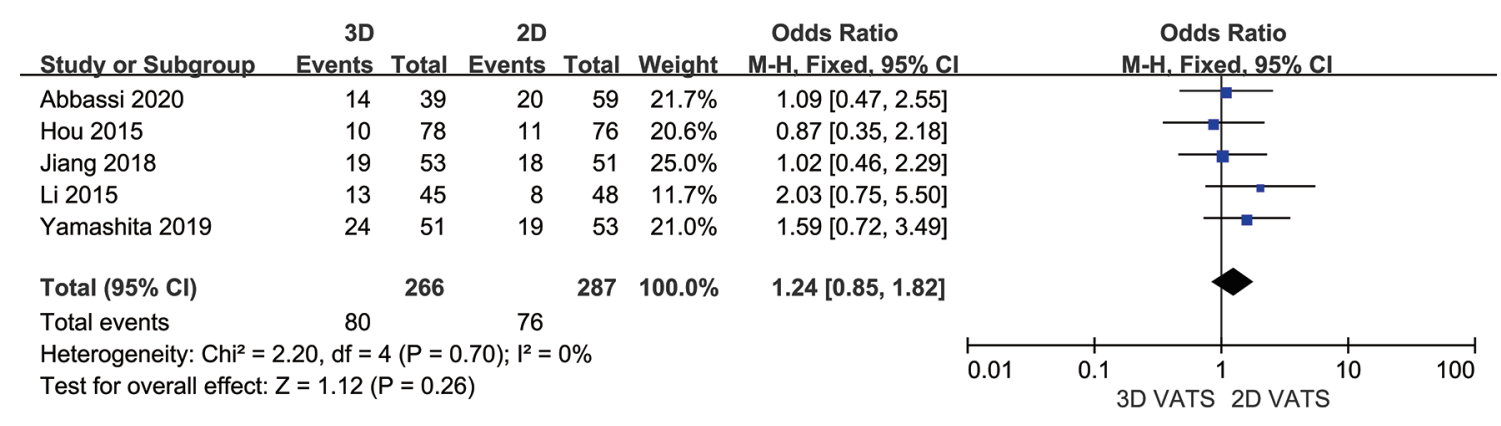

Figure 8 Forest plot of the role of OR and its 95\% CI of postoperative complications between 3D and 2D VATS group. OR, odds ratio; CI, confidence interval; VATS, video-assisted thoracic surgery. 
especially in deep narrow spaces of cleaning the recurrent laryngeal nerve lymph nodes, which leads to the prolongation of the learning curve. With the rapid development of thoracoscopic surgery techniques and instruments, 3D VATS has been used for patients of esophageal cancer. More precise and rapid dissection in narrow operation space becomes possible by means of restoring depth perception and spatial orientation $(24,26)$.

Our analysis suggested that the operation time in 3D VATS group was much shorter than that in 2D VATS group, and blood loss in 3D VATS group also decreased significantly compared with that in 2D VATS group. These findings are in line with the reported clinical benefits of implementing the 3D system, such as less blood loss, shorter operation time and shorter hospital stay (27-30). We believe that this is because under the $3 \mathrm{D}$ sense for operation of the instrument under $3 \mathrm{D}$ thoracoscope, the tissue is layered and the structure is exposed more clearly. So, it can provide a better depth field of vision when dissociating the esophagus and gastric curvature, thus reduce the practical time to flip and pull the surrounding tissue to expose the better visual field in 2D. Meanwhile, 3D can display the anatomical boundaries between the esophagus, stomach, lymph nodes with the surrounding tissues more fully and clearly, and therefore reduce the time wasted by accidental injury of thoracic duct, tracheal membrane, liver and splenic artery. The skeletonization of blood vessels makes it possible to dissociate anatomical esophagus and radically dissect lymph nodes. In addition, it is easier to expose the $3 \mathrm{D}$ dissection system of the left and right recurrent laryngeal nerve chain and peripheral lymph nodes, so as to avoid damage.

Lymph node status is regarded as a core prognostic index for esophageal cancer (31). The number of lymph nodes which were resected for esophageal cancer serves as a sign of radical resection as well as an independent predictor of survival (32). In our analysis no significant difference was found in the totality of lymph node dissection between 3D VATS group and 2D VATS group. The shorter operation time in 3D VATS group did not come at the cost of less lymph node dissection. Besides, in $3(12,18,19)$ of our studies, the postoperative drainage and postoperative tube in 3D VATS group took shorter time compared with those in 2D VATS group. This may be related to clearer vision under $3 \mathrm{D}$, clearer boundaries and less accessory injury during lymph node dissection. According to our experience, lymph nodes can be removed more completely as so-called "en-bloc", with a better view provided by $3 \mathrm{D}$. At the same time, the lymphatic vessels can be clearly displayed to electro-coagulate so that the postoperative exudation can be reduced. Otherwise, dissection of lymph nodes in regions of the left recurrent laryngeal nerve and aortic arch is not practical for open and 2D esophagectomy due to limited information on spatial depth, calculated from secondary spatial depth clues and the operator's rich experience (12). Particularly, in the lymph node dissection of the left recurrent laryngeal nerve and aortic arch, in the process of thoracotomy and 2D VATS, without information about spatial depth, it can only be speculated according to the 2D spatial information and the operator's experience, which is easy to cause false injury and increase the incidence of postoperative drainage and postoperative complications.

Finally, no significant difference was found in the incidence of post-operative complications between 3D VATS group and 2D VATS group. Therefore, the safety and rationality of 3D surgery have been verified. In addition, the transition from 2D VATS to 3D VATS is easy (33), and the learning curve of 3D VATS esophagectomy may be shorter than that of $2 \mathrm{D}$ or robot-assisted esophagectomy (34-36). Beginners have an ideal ability to control the lens head when they take part in six $3 \mathrm{D}$ endoscopic surgeries, while beginners need to participate in more than ten 2D endoscopic surgeries in the past to have an ideal ability to control the lens (18). It should be easier for beginners to change from $3 \mathrm{D}$ direct vision surgery to $3 \mathrm{D}$ VATS for esophageal cancer than to 2D.

\section{Limitations}

There were certain limitations in our study. For one thing, we made a meta-analysis of 5 cohort studies rather than RCTs, which was likely to lower the validity of the overall results. For another, considering the fact that two of the studies lacked in the original data of mean and standard deviation, we employed the "mean variance estimation" to estimate them, probably weakening the credibility of the results. Although the $\mathrm{P}$ value is small enough to show that there is a significant difference between $2 \mathrm{D}$ and $3 \mathrm{D}$ of operative time and blood loss. But there would be inherent bias because of the surgeon experience as the SD of operative time and blood loss is large. Therefore, much 
more study is needed to value the benefit of 3D VATS for esophageal cancer. Thus, in order to more accurately assess the safety and effectiveness of 3D VATS for esophageal cancer, high-quality randomized studies are needed to in the future study. In addition, none of the included studies mentioned whether 3D brings discomfort such as dizziness and headache, so the safety of $3 \mathrm{D}$ for surgery remains to be further confirmed.

\section{Conclusions}

According to this meta-analysis, we found that that $3 \mathrm{D}$ VATS for esophageal cancer shortens the operation time and lessens intraoperative blood loss without increasing the occurrence of postoperative complications and sacrificing the number of lymph node dissection. Consequently, we expect that $3 \mathrm{D}$ esophageal cancer surgery will be applied more widely in the future.

\section{Acknowledgments}

Funding: Our research is supported by the National Natural Science Foundation of China with grant/award number of 81402449 .

\section{Footnote}

Reporting Checklist: The authors have completed the PRISMA reporting checklist. Available at https://dx.doi. org/10.21037/tcr-21-644

Peer Review File: Available at https://dx.doi.org/10.21037/ tcr-21-644

Conflicts of Interest: All authors have completed the ICMJE uniform disclosure form (available at https://dx.doi. org/10.21037/tcr-21-644). The authors have no conflicts of interest to declare.

Ethical Statement: The authors are accountable for all aspects of the work in ensuring that questions related to the accuracy or integrity of any part of the work are appropriately investigated and resolved.

Open Access Statement: This is an Open Access article distributed in accordance with the Creative Commons Attribution-NonCommercial-NoDerivs 4.0 International
License (CC BY-NC-ND 4.0), which permits the noncommercial replication and distribution of the article with the strict proviso that no changes or edits are made and the original work is properly cited (including links to both the formal publication through the relevant DOI and the license). See: https://creativecommons.org/licenses/by-nc-nd/4.0/.

\section{References}

1. Mariette C, Dahan L, Mornex F, et al. Surgery alone versus chemoradiotherapy followed by surgery for stage I and II esophageal cancer: final analysis of randomized controlled phase III trial FFCD 9901. J Clin Oncol 2014;32:2416-22.

2. Takeuchi H, Miyata H, Gotoh M, et al. A Risk Model for Esophagectomy Using Data of 5354 Patients Included in a Japanese Nationwide Web-Based Database. Ann Surg 2014;260:259-66.

3. Herskovic A, Martz K, Al-sarraf M, et al. Combined Chemotherapy and Radiotherapy Compared with Radiotherapy Alone in Patients with Cancer of the Esophagus. N Engl J Med 1992;326:1593-8.

4. Cooper JS, Guo MD, Herskovic A, et al. Chemoradiotherapy of Locally Advanced Esophageal Cancer. JAMA 1999;281:1623-7.

5. Ozawa S, Tachimori Y, Baba H, et al. Comprehensive Registry of Esophageal Cancer in Japan, 2001. Esophagus 2009;6:95-110.

6. Tachimori Y, Ozawa S, Numasaki H, et al. Efficacy of lymph node dissection by node zones according to tumor location for esophageal squamous cell carcinoma. Esophagus 2016;13:1-7.

7. Cuschieri A. Endoscopic subtotal oesophagectomy for cancer using the right thoracoscopic approach. Surg Oncol 1993;2 Suppl 1:3-11.

8. Biere SS, van Berge Henegouwen MI, Maas KW, et al. Minimally invasive versus open oesophagectomy for patients with oesophageal cancer: a multicentre, open-label, randomised controlled trial. Lancet 2012;379:1887-92.

9. Nafteux P, Moons J, Coosemans W, et al. Minimally invasive oesophagectomy: a valuable alternative to open oesophagectomy for the treatment of early oesophageal and gastro-oesophageal junction carcinoma. Eur J Cardiothorac Surg 2011;40:1455-63; discussion 1463-64.

10. Luketich JD, Pennathur A, Franchetti Y, et al. Minimally invasive esophagectomy: results of a prospective phase II multicenter trial-the eastern cooperative oncology group 
(E2202) study. Ann Surg 2015;261:702-7.

11. Kauppi J, Rsnen J, Sihvo E, et al. Open versus minimally invasive esophagectomy: clinical outcomes for locally advanced esophageal adenocarcinoma. Surg Endosc 2015;29:2614-9.

12. Li Z, Li J, Qin X, et al. Three-dimensional vs twodimensional video assisted thoracoscopic esophagectomy for patients with esophageal cancer. World J Gastroenterol 2015;21:10675-82.

13. Zheng CH, Lu J, Zheng HL, et al. Comparison of 3D laparoscopic gastrectomy with a 2D procedure for gastric cancer: A phase 3 randomized controlled trial. Surgery 2018;163:300-4.

14. Lui MW, Cheung VY. Three-dimensional versus twodimensional laparoscopy for ovarian cystectomy: a prospective randomised study. Hong Kong Med J 2018;24:245-51.

15. Panic N, Leoncini E, de Belvis G, et al. Evaluation of the endorsement of the preferred reporting items for systematic reviews and meta-analysis (PRISMA) statement on the quality of published systematic review and metaanalyses. PLoS One 2013;8:e83138.

16. Wells G, Shea BJ, O'Connell D, et al. The Newcastle-Ottawa Scale (NOS) for Assessing the Quality of Non-Randomised Studies in Meta-Analyses. Proceedings of the Symposium on Systematic Reviews. Beyond the Basics, 2014.

17. Luo D, Wan X, Liu J, et al. How to estimate the sample mean and standard deviation from the sample size, median, extremes or quartiles? Chinese Journal of Evidence-Based Medicine 2017;17:1350-6.

18. Hou Y, Guo W, Yang Z, et al. Comparative study of 3D thoracoscopic esophagectomy versus 2D thoracoscopic esophagectomy for esophageal carcinoma. Zhonghua Wei Chang Wai Ke Za Zhi 2015;18:889-92.

19. Jiang J, Yu X, Geng G, et al. PS01.130: Video-assisted thoracoscope 3D and 2D mode comparative analysis for esophageal chest surgery. Dis Esophagus 2018;31:86-7.

20. Yamashita K, Mine S. The usefulness of three-dimensional video-assisted thoracoscopic esophagectomy in esophageal cancer patients. Esophagus 2019;16:272-7.

21. Abbassi O, Patel K, Jayanthi NV. Three-Dimensional vs Two-Dimensional Completely Minimally Invasive 2-Stage Esophagectomy With Intrathoracic Hand-Sewn Anastomosis for Esophageal Cancer: Comparison of Intraand Postoperative Outcomes. Surg Innov 2020. [Epub ahead of print]. doi:10.1177/1553350620972546

22. Ruurda JP, van Vroonhoven TJ, Broeders IA. Robot- assisted surgical systems: a new era in laparoscopic surgery. Ann R Coll Surg Engl 2002;84:223-6.

23. Heemskerk J, Zandbergen R, Maessen J, et al. Advantages of advanced laparoscopic systems. Surg Endosc 2006;20:730-3.

24. Wilhelm D, Reiser S, Kohn N, et al. Comparative evaluation of HD 2D/3D laparoscopic monitors and benchmarking to a theoretically ideal 3D pseudodisplay: even well-experienced laparoscopists perform better with 3D. Surg Endosc 2014;28:2387-97.

25. Hubber JW, Taffinder N, Russell RC, et al. The effects of different viewing conditions on performance in simulated minimal access surgery. Ergonomics 2003;46:999-1016.

26. Feng X, Morandi A, Boehne M, et al. 3-Dimensional (3D) laparoscopy improves operating time in small spaces without impact on hemodynamics and psychomental stress parameters of the surgeon. Surg Endosc 2015;29:12319. Erratum in: Surg Endosc. 2015 May;29(5):1240. doi: 10.1007/s00464-015-4155-4.

27. Sørensen SM, Savran MM, Konge L, et al. Threedimensional versus two-dimensional vision in laparoscopy: a systematic review. Surg Endosc 2016;30:11-23.

28. Arezzo A, Vettoretto N, Francis NK, et al. The use of 3D laparoscopic imaging systems in surgery: EAES consensus development conference 2018. Surg Endosc 2019;33:3251-74.

29. Liang H, Liang $W$, Lei $Z$, et al. Three-Dimensional Versus Two-Dimensional Video-Assisted Endoscopic Surgery: A Meta-analysis of Clinical Data. World J Surg 2018;42:3658-68.

30. Fergo C, Burcharth J, Pommergaard H, et al. Threedimensional laparoscopy vs 2-dimensional laparoscopy with high-definition technology for abdominal surgery: a systematic review. Am J Surg 2017;213:159-70.

31. Veeramachaneni NK, Zoole JB, Decker PA, et al. Lymph node analysis in esophageal resection: American College of Surgeons Oncology Group Z0060 trial. Ann Thorac Surg 2008;86:418-21; discussion 421.

32. Peyre CG, Hagen JA, DeMeester SR, et al. The number of lymph nodes removed predicts survival in esophageal cancer: an international study on the impact of extent of surgical resection. Ann Surg 2008;248:549-56.

33. Kyriazis I, Özsoy M, Kallidonis P, et al. Integrating ThreeDimensional Vision in Laparoscopy: The Learning Curve of an Expert. J Endourol 2015;29:657-60.

34. Veronesi G. Robotic thoracic surgery: technical considerations and learning curve for pulmonary resection. 
Thorac Surg Clin 2014;24:135-41, v.

35. Storz P, Buess G, Kunert W, et al. 3D HD versus 2D HD: surgical task efficiency in standardised phantom tasks. Surg Endosc 2012;26:1454-60.

Cite this article as: Xin N, Ding X, Huang K, Wei R, Chen Z, Liu C, Fang Y, Xu Z, Tang H. Three-dimension versus twodimension video-assisted thoracoscopic surgery for esophageal cancer: a meta-analysis. Transl Cancer Res 2021;10(7):3448-3457. doi: 10.21037/tcr-21-644
36. Park A, Lee G, Seagull F, et al. Patients benefit while surgeons suffer: an impending epidemic. J Am Coll Surg 2010;210:306-13. 